\title{
SYNOPSIS
}

News - Analysis - Practice

Drug Database

\section{Adverse reaction database has minimal information}

Critics charge that Health Canada's recently minted Canadian Adverse Drug Reaction Information System (CADRIS) is user-unfriendly and downright uninformative, in that it doesn't allow physicians or patients to determine the likely incidence of adverse drug reactions

Health Canada stands behind its strategy in presenting these data.

The inclusion of measures of drug usage, or another form of common denominator, would have invariably invited comparisons of the relative risk of various drugs, leading to unwarranted conclusions about safety and the inappropriate use of CADRIS as a prescriptive tool, says Heather Sutcliffe, director of Health Canada's marketed health products safety and effectiveness information division.

"It's well known that adverse reactions are underreported and that patient exposure is not known. And also adverse reaction reports reflect the suspicions of those who report them [whereas] a cause-and-effect relationship has not been established in the majority of cases. In general, because of the nature of the adverse reaction information, quantitative comparisons of health product safety can not be made from the data."

Such are the realities of CADRIS, unveiled and hailed in late May by federal Health Minister Ujjal Dosanjh "as a major step forward in Health Canada's transparency agenda."

The aim was to make the reports of more than 170000 ad- verse reactions reported to Health Canada since 1965 "more available and more accessible," Sutcliffe says.

But consumer safety groups say that "shame" and "embarrassment," rather than transparency, were at the root of CADRIS' release. Health Canada reluctantly released the database after the CBC had acquired it through the Access to Information Act and posted it online, argue Colleen Fuller and Terence Young, who chair the advocacy groups PharmaWatch and Drug Safety Canada respectively.

They charge that CADRIS' cumbersome nature, confusing parameters and total absence of comparative tools, such as the ability to search by disease or class of drugs, make it clear Health Canada only wanted to present a bare bones compendium of adverse reactions, rather than a useful tool for making informed drug decisions. "This is a baby step toward where we need to go," says Fuller.

Despite its limitations, CADRIS could have some utility, says Dr. Joel Lexchin, a professor of health policy at York University. It could, for example, help a physician contemplating the use of rarer drugs for a patient in a specific age or gender group.

But CADRIS must become far more amenable to sophisticated analysis before it can become a truly useful prescriptive tool or a method to ascertain whether or not particular classes of new drugs are causing adverse reactions, Lexchin adds. "There's lots more to be done."

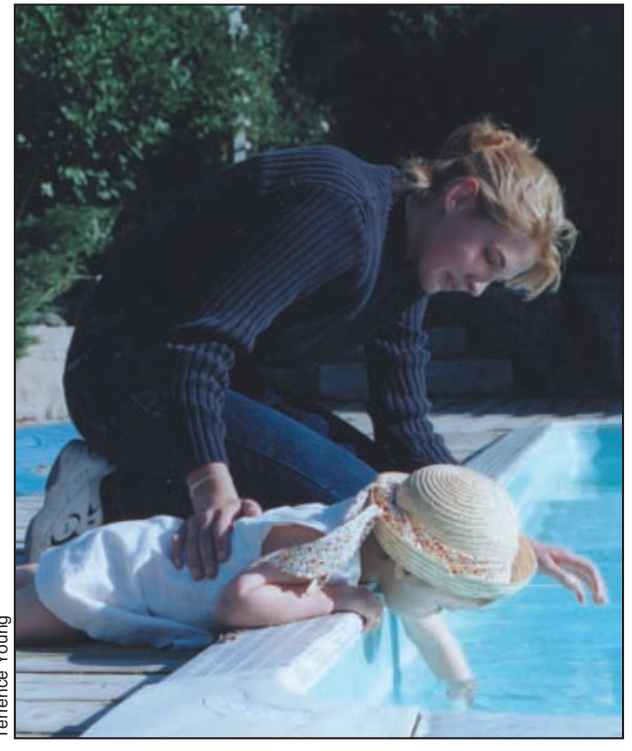

Vanessa Young's death from an adverse drug reaction sparked a call for action.

That level of utility isn't likely to be achieved unless mandatory reporting is introduced for all health professionals and management of the system is turned over to an independent drug safety agency, Young says.

An estimated 10000 Canadians die annually from adverse reactions but only $1 \%$ to $5 \%$ of such reactions are reported to health authorities. It's clear mandatory reporting is absolutely essential to promoting drug safety and providing early warning signals about drugs, Young says.

"But the only way it's going to happen is if some minister of health or the prime minister says this is the way we're going to clean this up and reduce the number of deaths." - Wayne Kondro, Ottawa 